\title{
A NEW LOG-NORMAL DISTRIBUTION
}

\section{TIANHAO WU}

Department of Statistics

Yale University

New Haven, CT 06511

USA

e-mail: tianhao.wu@yale.edu

\begin{abstract}
Log-normal distribution is ubiquitous in natural and social science by its simple form and high relation to normal distribution. However, oftentimes log-normal realizations can be dependent, so the application of $n$-dimensional log-normal is important and useful. In this paper, we develop a new form of log-normal distribution, whose marginals are proved to be independent of the power parameter $\theta$. In addition, this new distribution has special properties such as zero lower tail dependence and equidistribution which can be founded insightful for future research.
\end{abstract}

\section{Introduction}

One-dimensional log-normal distribution is ubiquitous in natural and social science by its simple form and high relation to normal distribution, whose cumulative probability distribution function is defined as $\Phi\left(\frac{\ln (X)-\mu}{\sigma}\right)$. Intrigued by the dependence among different realizations of log-normal, bivariate as well as high-dimensional log-normal attracts researchers among different disciplines.

2010 Mathematics Subject Classification: 62E10.

Keywords and phrases: log-normal, $n$-dimension, copula, dependence.

Received August 15, 2016; Revised September 7, 2016

(C) 2016 Scientific Advances Publishers 
The most-widely used multivariate log-normal random variate is defined as $Y=\prod X_{i}$, where $X_{i} \sim L N\left(\mu_{i}, \sigma_{i}^{2}\right)$, so $Y$ is distributed as

$$
Y \sim L N\left(\sum_{i} \mu_{i}, \sum_{i} \sigma_{i}^{2}\right)
$$

That implied that each marginal has different mean and variance. Furthermore, multivariate log-normal is founded to perfectly describe many natural and social phenomenon. Yue [6] uses bivariate log-normal distribution to represent multiple episodic storm events at the Motoyama meteorological observation station in Japan. Yerel and Konuk [4] applied similar distribution to study Beylikova magnesite ore deposit impurities in Eskisehir (Turkey). And Yue [5] fits the flood-frequency data also by a bivariate form of log-normal distribution.

In the past studies, many work concentrate on the theoretical aspects of the existing multivariate form mentioned above, such like the dependence measure (Nalbach-Leniewska [1]). Nevertheless, attempts to extend log-normal is rarely seen, among them Shaha and Bhavsar [3] is an example to construct a two piece multivariate distribution.

In this paper, we develop a new version of $n$-dimensional log-normal distribution which is a natural extension of the one-dimensional one. After proving it is a proper distribution, some of its properties are given. One of the promising properties is that its margins are distributed lognormal with same location and scale parameters, and its lower tail dependence attains zero which indicates that a realization of $\mathbf{X}$ has small probability to be a vector of 0 .

\section{2. $n$-Dimensional Log-Normal Distribution}

In this section, we will present the new version of log-normal distribution and show it is indeed a proper probability measure. Suppose $\mathbf{X}=\left\{X_{1}, X_{2}, \ldots, X_{n}\right\}$ is a vector of random variables and we define the new form of log-normal distribution whose jointly cumulative probability distribution function is 


$$
F(\mathbf{X})= \begin{cases}\Phi\left(\frac{\ln \left(\sum_{i} X_{i}^{\theta}\right)^{1 / \theta}-\mu}{\sigma}\right), & \text { if } \mathbf{X}>0 \\ 0, & \text { otherwise }\end{cases}
$$

where $\Phi$ denotes the cumulative probability function of a standard normal random variable, $\theta<0, \mu>0$, and $\sigma>0$. Also, $\mu$ and $\sigma$ are both finite. In this section, we first prove that it is indeed a proper $n$-dimensional distribution function if it satisfies the following definition ${ }^{1}$.

Definition 2.1. An $n$-dimensional distribution function is a function $F$ with domain $\mathbf{R}^{n}$ such that (i) $F(\mathbf{t})=0$ for all $\boldsymbol{t}$ in $\mathbf{R}^{n}$ such that $t_{k} \rightarrow-\infty$ for at least one $k$, and $F(\infty, \infty, \ldots, \infty)=1$; (ii) $F$ is $n$-increasing.

We then turn to prove that the distribution defined in Equation (1) follows the above two conditions.

Proof. (i) If $t_{k} \rightarrow-\infty$ for at least one $k, F(\mathbf{t})=0$ by our definition. And

$$
\lim _{\mathbf{t} \rightarrow \infty} \frac{\ln \left(\sum_{i} t_{i}^{\theta}\right)^{1 / \theta}-\mu}{\sigma}=\frac{\frac{1}{\theta} \ln 0-\mu}{\sigma} \rightarrow \infty,
$$

by the negativity of $\theta$. Thus,

$$
\lim _{\mathbf{t} \rightarrow \infty} \Phi\left(\frac{\ln \left(\sum_{i} t_{i}^{\theta}\right)^{1 / \theta}-\mu}{\sigma}\right)=1 .
$$

Hence condition (i) is satisfied.

${ }^{1}$ This definition comes from Page 45 on Nelsen [2]. 
(ii) For two vectors $\mathbf{a}$ and $\mathbf{b}$ such that $\mathbf{a} \leq \mathbf{b}$, if $V_{F}([\mathbf{a}, \mathbf{b}])=\Delta_{a_{n}}^{b_{n}} \cdots \Delta_{a_{1}}^{b_{1}}$ $F(\mathbf{X}) \leq 0$, we say that $F$ is $n$-increasing. Note that

$$
\begin{aligned}
& \lim _{\mathbf{t} \rightarrow 0} \frac{\ln \left(\sum_{i} t_{i}^{\theta}\right)^{1 / \theta}-\mu}{\sigma} \rightarrow-\infty, \\
& \lim _{\mathbf{t} \rightarrow 0} \Phi\left(\frac{\ln \left(\sum_{i} t_{i}^{\theta}\right)^{1 / \theta}-\mu}{\sigma}\right)=0 .
\end{aligned}
$$

So $F(\mathbf{X})$ is continuous in $\left(X_{1}, X_{2}, \ldots, X_{n}\right)$. It's obvious that the following inequality holds:

$$
\frac{\partial^{n} F(\mathbf{X})}{\partial X_{1} \partial X_{2} \ldots \partial X_{n}}>0
$$

Therefore, the condition of $n$-increasing is satisfied.

By presenting the above proof, we have shows that the new lognormal we defined is indeed a proper $n$-dimensional probability distribution.

As a matter of fact, the distribution in this paper has standard lognormal margins. Suppose $F\left(X_{i}\right)$ is the cumulative probability distribution function of $X_{i}$, then we can deduce its form by the following steps:

$$
\begin{aligned}
F\left(X_{i}\right) & =\lim _{\mathbf{X}_{-i} \rightarrow \infty} F(\mathbf{X}) \\
& =\Phi\left(\frac{\frac{1}{\theta} \ln X_{i}^{\theta}-\mu}{\sigma}\right) \\
& =\Phi\left(\frac{\ln X_{i}-\mu}{\sigma}\right),
\end{aligned}
$$

where $\mathbf{X}_{-i}$ represents $\left\{X_{1}, . ., X_{i-1}, X_{i+1}, \ldots, X_{n}\right\}$. And that is exactly the one-dimensional log-normal distribution with location parameter $\mu$ 
and scale parameter $\sigma$. Hence, we claim that the marginal distribution of $X_{i}$ is distributed log-normal in our case.

\section{Properties}

In this section, we show some properties of the new log-normal distribution which can be used for future research.

\subsection{Density}

We can see the jointly cumulative distribution function takes the following form when $\mathbf{X}>0$ :

$$
F(\mathbf{X})=\int_{-\infty}^{t} \frac{1}{\sqrt{2 \pi}} \exp \left(-\frac{m^{2}}{2}\right) d m
$$

where $t=\frac{\ln \left(\sum_{i} X_{i}^{\theta}\right)^{1 / \theta}-\mu}{\sigma}$, by shifting the integral interval we can get

$$
F(\mathbf{X})=\int_{-\infty}^{\ln \left(\sum_{i} X_{i}^{\theta}\right)^{1 / \theta}} \frac{1}{\sqrt{2 \pi \sigma}} \exp \left(-\frac{(m-\mu)^{2}}{2 \sigma^{2}}\right) d m .
$$

We take the partial derivative w.r.t. $X_{j}$, we get

$$
F_{j}(\mathbf{X})=\frac{X_{j}^{\theta-1}}{\sum_{i} X_{i}^{\theta} \sqrt{2 \pi \sigma}} \exp \left(-\frac{\left(\ln \left(\sum_{i} X_{i}^{\theta}\right)^{1 / \theta}-\mu\right)^{2}}{2 \sigma^{2}}\right),
$$

which is the marginal density function of $X_{j}$ and by letting $n=1$, it's easy to see the density above is exactly that of univariate log-normal distribution. Taking high-order partial derivatives gets us the joint density, however, it's quite complicated. If we focus on the bivariate case, the following stands for the cumulative distribution function: 


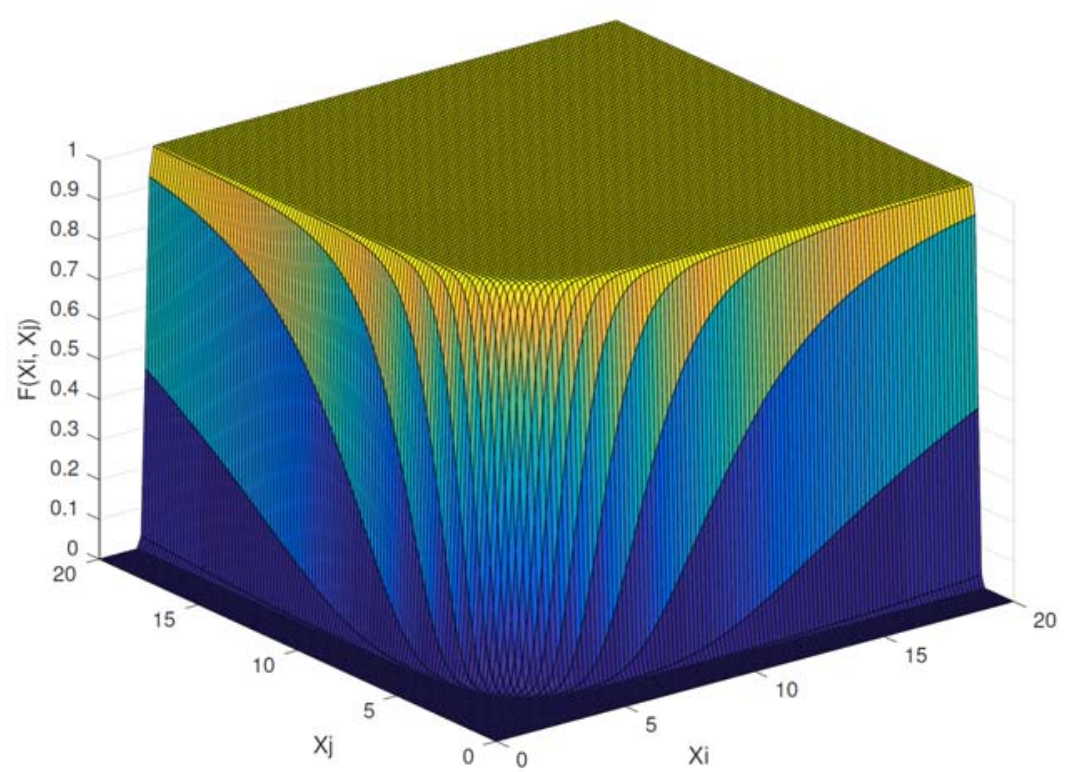

Figure 1. CDF of bivariate log-normal.

\subsection{Order statistics}

Define the $n$-order statistics of $\mathbf{X}=\left(X_{1}, X_{2}, \ldots, X_{n}\right)$ as $X_{(n)}$, then the distribution of which is

$$
\begin{aligned}
F\left(X_{(n)} \leq x\right) & =F\left(X_{1} \leq x, X_{2} \leq x, \ldots, X_{n} \leq x\right) \\
& =\Phi\left(\frac{\ln \left(n x^{\theta}\right)^{1 / \theta}-\mu}{\sigma}\right) \\
& =\Phi\left(\frac{\ln x+\frac{1}{\theta} \ln n-\mu}{\sigma}\right)
\end{aligned}
$$

so the maximum of $X_{i}$ is distributed log-normal with location parameter $\mu-\frac{1}{\theta} \ln n$ and scale parameter $\sigma$. Recall the margins, so the distribution of the maximum always has larger mean but same variation. Moreover, the bigger the number of variables in the vector $\mathbf{X}$, the bigger the mean. 


\subsection{Equidistribution}

From the definition, we know that any subset of $\mathbf{X}$ is distributed the same from of the new log-normal, so we conclude that $F(\mathbf{X})$ satisfies the equidistribution condition, i.e.,

$$
F\left(X_{1}, \ldots, X_{n-1}\right) \stackrel{d}{=} F\left(X_{2}, \ldots, X_{n}\right) .
$$

In fact, the distribution functions have the same forms if the dimension are same.

\subsection{Copula}

The following represents the copula of the $n$-dimensional log-normal distribution:

$$
C\left(u_{1}, u_{2}, \ldots, u_{n}\right)=\Phi\left(\frac{\frac{1}{\theta} \ln \left(\sum_{i} \exp \left(\theta \Phi^{-1}\left(u_{i}\right) \sigma+\mu \theta\right)\right)-\mu}{\sigma}\right), \mathbf{u} \in[0,1]^{n}
$$

Define an inverse function $\varphi^{-1}(u)$ as $\varphi^{-1}(u)=\Phi\left(\frac{\ln u^{1 / \theta}-\mu}{\sigma}\right)$, then $\varphi(u)=\exp \left(\theta \sigma \Phi^{-1}(u)+\mu \theta\right)$. Then the $n$-dimensional copula can be seen as

$$
C\left(u_{1}, u_{2}, \ldots, u_{n}\right)=\varphi^{-1}\left(\sum_{i} \varphi\left(u_{i}\right)\right)
$$

We claim it is an Archimedean copula, whose definition is as follows.

Definition 3.4.1. If $\varphi$ is a continuous strictly decreasing function from $I$ to $[0, \infty]$ such that $\varphi(1)=0$ and $\varphi(0)=\infty$. Then $C$ is an Archimedean $n$-copula if and only if $\varphi^{-1}$ is completely monotonic on $[0, \infty)$.

Theorem 1. The n-copula for multivariate log-normal distribution is an Archimedean copula. 
Proof. (i) As $\Phi$ is the cumulative probability distribution function for stand normal random variable so $\Phi$ is strictly increasing, as is $\Phi^{-1}$. Then by the negativity of $\theta$ and positivity of $\sigma$, we know that $\varphi(u)$ is strictly decreasing in $u$.

(ii) The domain of $\varphi$ is $[0,1]$ by its definition and $\varphi(u) \leq 0$ by the property of exponential function. Hence $\varphi$ is mapping from $I$ to $[0, \infty]$.

(iii) $\Phi^{-1}(1) \rightarrow \infty$, thus $\theta \sigma \Phi^{-1}(1)+\mu \theta \rightarrow-\infty$ and $\varphi(1)=0 . \quad$ In addition, $\Phi^{-1}(0) \rightarrow-\infty$, thus $\theta \sigma \Phi^{-1}(0)+\mu \theta \rightarrow \infty$ and $\varphi(0)=1$.

(iv) The complete monotonicity of $\varphi^{-1}$ is pinned down to show that of $\ln u^{1 / \theta}$. It's easy to show that $(-1)^{k} f^{(k)} \geq 0$ for $x>0$ and $k=0,1,2$, $3, \ldots, f(u)=\ln u^{1 / \theta}$. This completes the proof.

\subsection{Tail dependence}

By the copula above, we are able to calculate the following conditional probability:

$$
T_{u}=\operatorname{Pr}\left(F\left(X_{i}\right) \leq u \mid F\left(X_{j}\right) \leq u\right)=\frac{C(u, u)}{u}=\frac{\Phi\left(\Phi^{-1}(u)+\theta^{-1} \sigma^{-1} \ln 2\right)}{u},
$$

so the lower tail dependence is

$$
\lim _{u \downarrow 0} T_{u}=\lim _{u \downarrow 0}\left(\frac{\Phi\left(\Phi^{-1}(u)+\theta^{-1} \sigma^{-1} \ln 2\right)}{u}\right)=0 .
$$

Proof. By L'Hospital's rule, we have

$$
\lim _{u \downarrow 0} \frac{\Phi\left(\Phi^{-1}(u)+\theta^{-1} \sigma^{-1} \ln 2\right)}{u}=\lim _{u \downarrow 0} \frac{1}{\sqrt{2 \pi}} \frac{\partial \Phi^{-1}(u)}{\partial u} \exp \left(-\frac{t^{2}}{2}\right),
$$

where $t=\Phi^{-1}(u)+\theta^{-1} \sigma^{-1} \ln 2$. By the property of inverse function, moreover, 


$$
\begin{aligned}
\lim _{u \downarrow 0} \frac{1}{\sqrt{2 \pi}} \frac{\partial \Phi^{-1}(u)}{\partial u} \exp \left(-\frac{t^{2}}{2}\right) & =\lim _{u \downarrow 0} \frac{1}{\sqrt{2 \pi}} \exp \left(-\frac{t^{2}}{2}\right) \frac{1}{\Phi^{\prime}\left(\Phi^{-1}(u)\right)} \\
& =\lim _{u \downarrow 0} \exp \left(-\frac{t^{2}}{2}\right) \exp \left(\frac{t^{\prime 2}}{2}\right),
\end{aligned}
$$

where $t^{\prime}=\Phi^{-1}(u)$. Simplifying it we get

$$
\lim _{u \downarrow 0} \exp \left(-\frac{t^{2}}{2}\right) \exp \left(\frac{t^{\prime 2}}{2}\right)=0 .
$$

That means if $X_{j}$ is pretty small, then $X_{i}$ cannot be small, or equivalently, $X_{i}$ has probability 0 to be very small.

\subsection{Conditional probability}

Recall the marginal density in the Subsection 3.1.

$$
F_{1}(\mathbf{X})=\frac{X_{j}^{\theta-1}}{\sum_{i} X_{i}^{\theta} \sqrt{2 \pi \sigma}} \exp \left(-\frac{\left(\ln \left(\sum_{i} X_{i}^{\theta}\right)^{1 / \theta}-\mu\right)^{2}}{2 \sigma^{2}}\right)
$$

which in fact has another interpretation,

$$
\operatorname{Pr}\left(X_{1} \leq x_{1}, \ldots, X_{j}=x_{j}, \ldots, X_{n} \leq x_{n}\right)=\frac{\partial F(\mathbf{X})}{\partial x_{j}} .
$$

Thus

$\operatorname{Pr}\left(X_{1} \leq x, \ldots, X_{j}=x, \ldots, X_{n} \leq x\right)=\frac{1}{n x \sqrt{2 \pi \sigma}} \exp \left(-\frac{\left(\frac{1}{\theta} \ln \left(n x^{\theta}\right)-\mu\right)^{2}}{2 \sigma^{2}}\right)$,

which is $\operatorname{Pr}\left(\max _{i} X_{i}=x \bigcap \arg \max _{i} X_{i}=j\right)$. Integrating the above probability from $x$ to $\infty$, 


$$
\begin{aligned}
\int_{x}^{\infty} \frac{1}{n x \sqrt{2 \pi \sigma}} \exp \left(-\frac{\left(\frac{1}{\theta} \ln \left(n x^{\theta}\right)-\mu\right)^{2}}{2 \sigma^{2}}\right) \\
\quad=\int_{x}^{\infty} \frac{1}{n x \sqrt{2 \pi \sigma}} \exp \left(-\frac{\left(\ln (x)+\theta^{-1} \ln (n)-\mu\right)^{2}}{2 \sigma^{2}}\right),
\end{aligned}
$$

whose integrating factor is the density of log-normal distribution with location parameter $\mu-\theta^{-1} \ln (n)$ and scale parameter $\sigma$. So the integration is

$$
1-\Phi\left(\frac{\ln (x)+\theta^{-1} \ln (n)-\mu}{\sigma}\right),
$$

which is $\operatorname{Pr}\left(\max _{i} X_{i} \geq x \cap \arg \max _{i} X_{i}=j\right)$. Furthermore, we have

$$
\begin{aligned}
\operatorname{Pr}\left(\max _{i} X_{i} \geq x\right) & =\sum_{j} \operatorname{Pr}\left(\max _{i} X_{i} \geq x \cap \arg \max _{i} X_{i}=j\right) \\
& =n-n \Phi\left(\frac{\ln (x)+\theta^{-1} \ln (n)-\mu}{\sigma}\right) .
\end{aligned}
$$

So the condition probability is

$$
\begin{gathered}
\operatorname{Pr}\left(\arg \max _{i} X_{i}=j \mid \max _{i} X_{i} \geq x\right)=\frac{\operatorname{Pr}\left(\max _{i} X_{i} \geq x \cap \arg \max _{i} X_{i}=j\right)}{\operatorname{Pr}\left(\max _{i} X_{i} \geq x\right)} \\
=\frac{1}{n} .
\end{gathered}
$$

Also note that $\operatorname{Pr}\left(\arg \max _{i} X_{i}=j\right)=\frac{1}{n}$ which is trivial to prove. So the probability that maximizer of $X_{i}$ is $X_{j}$ given a threshold of the maximum only depends on the number of elements in $\mathbf{X}$.

\subsection{The role of $\theta$}

We are interested in the role of $\theta$, we will explore it of special cases in this section. First note that by the unboundedness of $\ln (\cdot)$ function, 
taking derivative of $F(\mathbf{X})$ w.r.t. $\theta$ produces unsigned result. However, if we focus on $\frac{\partial F(x, x, \ldots, x)}{\partial \theta}$, whose sign only depends on $\frac{\partial\left(n x^{\theta}\right)^{1 / \theta}}{\partial \theta}$,

$$
\frac{\partial\left(n x^{\theta}\right)^{1 / \theta}}{\partial \theta}=-x n^{1 / \theta} \ln (n) \frac{1}{\theta^{2}}<0 \text { since } n \geq 1,
$$

so $\frac{\partial F(x, x, \ldots, x)}{\partial \theta}<0$, the larger $\theta$, the smaller the probability that all $X$ fall down to the same interval. Additionally,

$$
\lim _{\theta \rightarrow 0}\left(\sum_{i} X_{i}^{\theta}\right)^{1 / \theta} \rightarrow \infty, \text { for } i>1
$$

so

$$
\lim _{\theta \rightarrow 0} F(\mathbf{X}) \rightarrow 1, \text { for } i>1,
$$

thus the joint probability converges to 1 when $\theta$ goes to 0 .

As a matter of fact, the function $f(\mathbf{x})=\left(\sum_{i} x_{i}^{\theta}\right)^{1 / \theta}$ is called constant elasticity of substitution (CES) function and has a variety of applications in Economics and Engineering. To see this, we can prove that

$$
f(t \mathbf{x})=t f(\mathbf{x}),
$$

so if $\mathbf{X} \sim F(\mathbf{X} ; \mu, \sigma), a \mathbf{X} \sim F(\mathbf{X} ; \mu+\ln (a), \sigma)$. And $\theta$ is a measure of substitutability and the bigger the $\theta$, the bigger the substitutability of $X_{i}$. Accordingly, $\theta$ plays the role of measuring the across differences among $\left(X_{1}, X_{2}, \ldots, X_{n}\right)$ to some extent.

\subsection{Discussion}

The properties proved above can be potentially used in future research. For example, its equidistribution property can draw attention into fitting a sequence of events whose every subset is distributed equivalently log-normal. And by its copula, one can easily compute the dependence between random variables. Furthermore, as its tail dependence is 0 , the new log-normal distribution is likely to be applied to some real data in which not all values can be very small. 


\section{Conclusion}

In this paper, we develop a new form of $n$-dimensional log-normal distribution which depends on the shape parameter $\theta$ and scale parameter $\sigma$. This new log-normal distribution is proved to have stand log-normal margins, indicating that each $X_{i}$ is distributed univariate log-normal. Furthermore, some properties such as its copula, equidistribution, tail dependence, etc. are given. Readers should see this work as a framework and some more research can be done regarding this new form of log-normal, for example, the application of that to some real data and see the ability of this distribution of describing random events.

\section{Acknowledgement}

The author thanks the anonymous referee for his/her valuable comments. All errors are my own.

\section{References}

[1] A. Nalbach-Leniewska, Measures of dependence of the multivariate log-normal distribution, Series Statistics 10(3) (1979), 381-387.

[2] R. B. Nelsen, An Introduction to Copulas, Springer Series in Statistics, 2006.

[3] P. Shaha and C. D. Bhavsar, Two-piece multivariate log-normal distribution, International Journal of Sciences: Basic and Applied Research 15(1) (2014), 164-170.

[4] S. Yerel and A. Konuk, Bivariate log-normal distribution model of cutoff grade impurities: A case study of magnesite ore deposit, Scientific Research and Essay 4(12) (2009), 1500-1504.

[5] S. Yue, The bivariate log-normal distribution to model a multivariate flood episode, Hydrological Processes 14 (2000), 2575-2588.

[6] S. Yue, The bivariate log-normal distribution for describing joint statistical properties of a multivariate storm event, Environmetrics 13 (2002), 811-819. 